\title{
Bundesverdienstkreuz für Prof. Dr. Friedrich Wilhelm Schwartz
}

Prof. Dr. Friedrich Wilhelm Schwartz, Mitglied des Beirates der Zeitschrift „Das Gesundheitswesen“, wurde für seine außergewöhnlichen Verdienste um das allgemeine Wohl mit dem Verdienstkreuz am Bande des Verdienstordens der Bundesrepublik Deutschland geehrt. Der Verdienstorden, 1951 von Bundespräsident Theodor Heuss gestiftet, wird vergeben für Leistungen, die auf politischem, wirtschaftlich-sozialem und geistigem Gebiet, aber auch im Bereich der sozialen, karitativen und mitmenschlichen Hilfe erbracht werden.

Der Verdienstorden wurde vom Bundespräsidenten Host Köhler verliehen und durch Staatssekretär Dr. Josef Lange, Niedersächsisches Ministerium für Wissenschaft und Kultus, an Prof. Schwartz überreicht. Die Laudatio würdigt insbesondere die Verdienste um die Förderung von Public Health in Deutschland und international sowie die wissenschaftliche Beratung in der Gesundheitspolitik, neben der großen Verdienste für die Medizinische Hochschule Hannover und die erfolgreiche Repräsentanz des eigenen Faches an der Hochschule und darüber hinaus. Die Schriftleitung der Zeitschrift „Das Gesundheitswesen“ und der Georg Thieme Verlag gratulieren Prof. Schwartz zu dieser hohen Auszeichnung.

In ihrem 53. Jahrgang (1991) wurde „Das öffentliche Gesundheitswesen“ auch Organ der Deutschen Gesellschaft für Sozialmedizin und Prävention (DGSMP). Als Fachvertreter wurde Prof. Schwartz Mitglied des Beirates der Zeitschrift, eine Aufgabe, die er bis heute wahrnimmt.

Der Titel der Zeitschrift wurde 1992 in „Das Gesundheitswesen“ geändert und der Untertitel um den Begriff Public Health erweitert. Zahlreiche Arbeiten zu diesem Thema bereichern seither das Spektrum der Zeitschrift. Diese wurde zu einer Drehscheibe für den Öffentlichen Gesundheitsdienst, den Medizinischen Dienst der Sozialversicherung und der im Hochschulbereich angesiedelten Public Health/Gesundheitswissenschaften. 1998 wurde „Das Gesundheitswesen“, insbesondere durch Anregung von Prof. Schwartz, auch Organ der Deutschen Gesellschaft für Public Health (DGPH).
Prof. Schwartz hat, in seiner Tätigkeit als Gutachter und Autor in der Zeitschrift und darüber hinaus, seine Positionen stets souverän vertreten mit hoher fachlicher Kompetenz und persönlichem Engagement, auch gegen vordergründig erfolgreichem Zeitgeist, der oft genug ängstliche Naturen zur Anpassung verleitet.

Die Theorie ist eine Vereinfachung der Realität (J. G.v. Goethe), das gilt besonders in der Medizin. F. W. Schwartz hat seine wissenschaftlichen Erkenntnisse immer wieder in die Realität der Gesundheitspolitik transportiert, ohne Rücksicht auf Beifall. Dementsprechend lautet seine Schlussfolgerung für die Sozialmedizin als wissenschaftliche Disziplin (Gesundheitswesen 1997; 59: 207-212), vorgetragen anlässlich der Verleihung der SalomonNeumann-Medaille:

- Sozialmedizin als bevölkerungsmedizinische Disziplin mit dem Ziel „Egalité“ in der Gesundheitsversorgung muss sich in die Politik einmischen.

- Sozialmedizin als empirische Wissenschaft muss Mythen und Vorurteile wissenschaftlich überprüfen und ggf. widerlegen.

Zum Verhältnis von Gesundheitsexperten und Staat (Gesundheitswesen 2006; 68: 70-80) schreibt er: „Aus gesellschaftlicher Sicht erwünscht ist dabei eine transparente Trennung zwischen dem interessengeleiteten und einem sachverpflichteten unabhängigen wissenschaftlichen Beratungsprozess ... In all diesen Prozessen ist ferner zukünftig eine stärkere (ebenfalls selbst und fremdkritisch zu reflektierende) Repräsentanz der Patienten und Konsumenten des Gesundheitswesens erwünscht, damit das „Gesundheitswesen“ keine Industrie oder Verwaltung für „dritte Zwecke“ jenseits ihrer Bedürfnisse und Präferenzen wird.“

Nur ein bequemer Zeitgenosse wollte Prof. Schwartz offensichtlich niemals sein, gerade deshalb ist er ein gefragter Gutachter, auch für die Zeitschrift „Das Gesundheitswesen“. Leser, Autoren und Schriftleitung danken ihm dafür.

Eine ausführliche Würdigung der Persönlichkeit und der Verdienste von Prof. Schwartz hat Ingo Strote, Dipl.-Päd., verfasst. 